\title{
The Pathogenicity of Chicken Pathogenic Escherichia coli Is Associated with the Numbers and Combination Patterns of Virulence-Associated Genes
}

\author{
Jingyu Wang1*, Pan Tang1, Dan Tan1, Liqin Wang1, Sandong Zhang1, Yuanhao Qiu ${ }^{1}$, \\ Rui Dong1, Wanhua Liu', Jingjing Huang1, Ting Chen'1, Juanjuan Ren'1, Cengshan Li', \\ Hung-Jen Liu ${ }^{2,3,4^{*}}$ \\ ${ }^{1}$ College of Veterinary Medicine, Northwest A\&F University, Yangling, China \\ ${ }^{2}$ Institute of Molecular Biology, National Chung Hsing University, Taiwan \\ ${ }^{3}$ Agricultural Biotechnology Center, National Chung Hsing University, Taiwan \\ ${ }^{4}$ Rong Hsing Research Center for Translational Medicine, National Chung Hsing University, Taiwan \\ Email: *wjingyu2004@126.com, "hjliu5257@nchu.edu.tw
}

Received 21 October 2015; accepted 6 December 2015; published 11 December 2015

Copyright @ 2015 by authors and Scientific Research Publishing Inc.

This work is licensed under the Creative Commons Attribution International License (CC BY).

http://creativecommons.org/licenses/by/4.0/

c) (i) Open Access

\begin{abstract}
Various virulence-associated genes or pathogenicity island are responsible for determining the pathogenicity of Escherichia coli strains. However, the correlation of the number and combination patterns of virulence-associated genes in Escherichia coli strains with their pathogenicity remains largely unknown. In this work, 581 chicken Escherichia coli strains were isolated from 1045 liver samples of dead chickens from 50 chicken farms at four provinces in China during 2007-2012. Based on the pathogenic test of SPF chickens, 320 chickens pathogenic Escherichia coli isolates were identified as highly $(n=193)$, intermediate $(n=98)$ and low pathogenic $(n=29)$ strains, respectively. Furthermore, the number of virulence genes in the 320 chicken pathogenic and 50 non-pathogenic Escherichia coli strains was examined. Our results reveal that thirteen virulence genes in Escherichia coli strains were detected, and all strains carried at least two or more than two virulence-associated genes. This study also suggests that highly pathogenic $E$. coli strains simultaneously carried at least 8 to13 virulence genes while intermediate pathogenic strains carried at least 5 to 8 virulence genes. The number of virulence-associated genes detected in highly pathogenic strains showed there were more significant differences than that in low pathogenic strains $(P<0.01)$. The detection rate of genes irp2, fyuA, and colV in high pathogenic strains was significantly higher than that in low and non-pathogenic strains $(P<0.01)$. Nine virulence-asso-
\end{abstract}

*Corresponding authors.

How to cite this paper: Wang, J.Y., et al. (2015) The Pathogenicity of Chicken Pathogenic Escherichia coli Is Associated with the Numbers and Combination Patterns of Virulence-Associated Genes. Open Journal of Veterinary Medicine, 5, $243-254$.

http://dx.doi.org/10.4236/ojvm.2015.512033 
ciated genes irp2, fyuA, iиcA, iucD, iutA, papC, iss, tsh, and colV were more often detected in highly and intermediate pathogenic $E$. coli strains. Taken together, our results provide evidences demonstrating that the pathogenicity of Escherichia coli strains is closely associated with the number and combination patterns of virulence-associated genes.

\section{Keywords}

\section{Avian Pathogenic Escherichia coli, Pathogenicity, Virulence-Associated Genes}

\section{Introduction}

The avian pathogenic Escherichia coli (APEC) infection of avian respiratory tract causes respiratory tract lesions and septicemia. This disease is referred to as air-sacculitis, pneumonitis, septicemia, and colibacillosis or coli septicemia. Colibacillosis is an important bacterial infectious disease against poultry industry [1]. In China, APEC causes broiler colibacillosis which results in serious economic losses to the poultry industry. Previous studies have suggested that virulence-associated genes and pathogenicity islands of bacteria play an important role in the pathogenicity of bacteria and that they are important parameters to clarify the mechanism of bacterial pathogenicity [2] [3]. To date, more than 25 virulence genes were detected in bacteria, such as the locus of enterocyte effacement (LEE), ColV and ColBM plasmid [4]-[7], high pathogenicity island (HPI) [8], and hemolysin A and temperature-sensitive hemagglutinin [9].

Various virulence-associated genes or pathogenicity island are responsible for determining the pathogenicity of Escherichia coli strains. In recent years, studies mainly focus on the types and distribution of virulence-associated genes as well as the relationship between distribution of virulence-associated genes and $\mathrm{O}$ serotypes. The aim of this study was to determine the relationship between pathogenicity of chicken pathogenic Escherichia coli and the number and combination patterns of virulence-associated genes. In the present study, 581 chicken Escherichia coli strains were isolated from 1045 liver samples of dead chickens from 50 poultry farms at Shaanxi, Henan, Hebei and Shanxi provinces in China during 2007-2012. Among 581 Escherichia coli strains tested, they were classified into high, intermediate, low pathogenic, and non-pathogenic groups. Among 320 pathogenic and 50 non-pathogenic Escherichia coli examined, 18 virulence-associated genes were identified by polymerase chain reaction (PCR) and sequence analysis. Our results reveal that the number and combination patterns of virulence-associated genes in Escherichia coli strains correlate with pathogenicity.

\section{Materials and Methods}

\subsection{Source of Chicken E. coli Isolates}

In the present study, a total of 581 chicken Escherichia coli strains were isolated from 1045 liver samples of sick and dead chickens from 50 chicken farms at Shaanxi, Henan, Hebei and Shanxi provinces in China during 2007-2012 (Table 1). Samples were collected from enlarge livers of sick and dead chicken in sterile condition. In the present study, 581 chicken Escherichia coli strains were cultured on MaConkey agar plates, and incubated at $37^{\circ} \mathrm{C}$ for $18 \mathrm{~h}$, and then single bacterial colony was picked up and purified. All 581 chicken $E$.coli strains were then sent to China Institute of Veterinary Drug Control for serotyping.

Table 1. Information concerning the sample collection and isolation.

\begin{tabular}{|c|c|c|c|c|}
\hline Place & No. flocks & Total No. samples & No. E.coli isolates & Distribution of O serotype \\
\hline Shaanxi & 18 & 310 & 165 & \multirow{5}{*}{$\begin{array}{c}\text { O1, O2, O6, O14, O16, O17, O22, } \\
\text { O38, O62, O70, O74, O78, O85, O88, } \\
\text { O93, O98, O101, O114, O115, } \\
\text { O123, O127, O128, O124, O161 }\end{array}$} \\
\hline Henan & 12 & 238 & 133 & \\
\hline Hebei & 9 & 255 & 145 & \\
\hline Shanxi & 11 & 242 & 138 & \\
\hline Total & 50 & 1045 & 581 & \\
\hline
\end{tabular}




\subsection{Pathogenic Test of SPF Chickens}

581 chicken Escherichia coli strains were cultured in LB broth at $37^{\circ} \mathrm{C}$ for $24 \mathrm{~h}$. All tested Escherichia coli strains were individually suspended in sterile saline at a concentration of $10^{8} \mathrm{CFU} / \mathrm{mL}$. In the pathogenic test, 581 groups of one-day-old White Leghorn specific-pathogen-free chickens supplied by Yangling Green Square Biological Engineering Co. (China) were used. Each group included five one-day-old White Leghorn specificpathogen-free chickens and was reared in separate cages with food and water. Each chicken was inoculated subcutaneously with $0.2 \mathrm{~mL}$ of Escherichia coli suspension $\left(1 \times 10^{8} \mathrm{CFU} / \mathrm{per} \mathrm{mL}\right)$. Deaths were recorded 4 times per day, and continuing for 7 days. Clinical signs of illness were recorded daily. The surviving chickens were killed at 7 day post-inoculation. The lesions were observed and bacteria were isolated and identified.

The pathogenicity of Escherichia coli strains was determined on the basis of lesions and mortalities as described previously [10]-[12]. The animal experiments were carried out in compliance with the regulations of the Guide for the Care and Use of Laboratory Animals prepared by the international animal welfare standards. All animal experiments in this study were approved by the committee on Research Animal Care of Northwest A and F University.

\subsection{PCR Amplification of Virulence-Associated Genes in Chicken E.coli Strains}

The different sets of primers (Table 2) used for PCR amplification were designed with Primer 5.0 software based on the previously published sequences [13] [14]. Primers were synthesized by Nanjing GenScript Biotechnology Co. (China).

All Escherichia coli strains and reference strains were grown on LB agar plates at $37^{\circ} \mathrm{C}$ overnight. Escherichia coli colonies were suspended in $500 \mu \mathrm{L}$ of deionized water and boiling for $10 \mathrm{~min}$, followed by chilling on ice for $5 \mathrm{~min}$ and centrifugation at 10,000 $\mathrm{xg}$ for $5 \mathrm{~min}$, the supernatant was used as the DNA templates for PCR amplification. The PCR mixture contained $10 \mu \mathrm{L}$ of $2 \times$ PCR Master mix (including $2 \times$ Taq DNA polymerase, $2 \times$ PCR Buffer and $2 \times$ dNTP mixture) (TaKaRa), $1 \mu \mathrm{L}$ of primer pair, $4 \mu \mathrm{L}$ of DNA template, and deionized water to a final volume of $25 \mu \mathrm{L}$. PCR was completed by an initial heat activation of $5 \mathrm{~min}$ at $95^{\circ} \mathrm{C}$; then 30 cycles of $30 \mathrm{~s}$ at $94^{\circ} \mathrm{C}, 30 \mathrm{~s}$ at Tm, and $45 \mathrm{~s}$ at $72^{\circ} \mathrm{C}$; and an extension of $10 \mathrm{~min}$ at $72^{\circ} \mathrm{C}$. PCR products were separated by size by $1 \%$ agarose gel electrophoresis along with DL2000 DNA markers and visualized after staining with ethidium bromide on a UV transilluminator. PCR products amplified from the virulence genes in Escherichia coli strains were sequenced and their sequences were compared with previously published sequences by using DNAStar software.

\subsection{Combination of Virulence-Associated Genes and Their Correlations with Pathogenicity}

Chi-square test were used to analyze the numbers and combination of virulence-associated genes in 320 chicken pathogenic and 50 non-pathogenic Escherichia coli strains and to analyze the numbers and combination of virulence-associated genes and their correlations with pathogenicity.

\section{Results}

\subsection{Pathogenic Test}

Among 581 Escherichia coli strains examined, 320 strains caused the death of chicken, suggesting that they are pathogenic Escherichia coli strains; 261 strains did not cause the death of chicken, suggesting that they are non-pathogenic Escherichia coli strains. Pathogenic test indicated that 320 chicken E. coli strains were classified into high , intermediate, and low pathogenicity groups based on the lesions and mortality of experimentally infected chickens [12]. Pathogenic test shows that the percentage of high pathogenic, intermediate pathogenic and low pathogenic strains was 60.3\% (193/320), 30.6\% (98/320), 9.1\% (29/320), respectively.

\subsection{Detection of Virulence-Associated Genes in Pathogenic Escherichia coli Strains by PCR and Sequence Analysis}

Among 18 virulence-associated genes examined, 13 virulence-associated genes $\operatorname{irp} 2$, fyuA, iucA, iucD, iutA, FimA, FimC, papC, iss, hlyA, tsh, colV, and colBM in 581 Escherichia coli strains were detected by PCR. The 
Table 2. Primers used in this study.

\begin{tabular}{|c|c|c|c|}
\hline Gene & Description & PCR products (bp) & Primer sequence $\left(5^{\prime} \text { to } 3^{\prime}\right)^{\mathrm{a}}$ \\
\hline \multicolumn{4}{|c|}{ pTJ100-related genes } \\
\hline \multirow{2}{*}{ iucA } & \multirow{2}{*}{ Aerobactin production } & \multirow{2}{*}{236} & F-CGCGAGCGGCTCATACAGG \\
\hline & & & R-TCGTCGGGCAGCGTTTCT \\
\hline \multirow{2}{*}{ iucD } & \multirow{2}{*}{ The aerogenes gene } & \multirow{2}{*}{400} & F-AGTTCTATCGCTTCCTTAC \\
\hline & & & R- GAGACCCAGTTTATTTCC \\
\hline \multirow{2}{*}{ iutA } & \multirow{2}{*}{ Ferric aerobactin receptor gene } & \multirow{2}{*}{424} & F-AACAAACCGATGATGAAACG \\
\hline & & & R-GTGCCAGCCTCAAACTCC \\
\hline \multirow{2}{*}{ iss } & \multirow{2}{*}{ Increased serum survival gene } & \multirow{2}{*}{756} & F-GTTCTCCGTCGGGCTACT \\
\hline & & & R-GCTCTGCGTGATGATGTT \\
\hline \multirow{2}{*}{ tsh } & \multirow{2}{*}{ Temperature-sensitive hemagglutinin gene } & \multirow{2}{*}{488} & F-ACGGTCAATAATGAACTCG \\
\hline & & & R-CAGGAATATGCACСТCCC \\
\hline \multicolumn{4}{|c|}{ Iron-related genes } \\
\hline \multirow{2}{*}{ irp2 } & \multirow{2}{*}{ Iron repressible gene } & \multirow{2}{*}{236} & F-CGCGAGCGGCTCATACAGG \\
\hline & & & R-TCGTCGGGCAGCGTTTCT \\
\hline \multirow{2}{*}{ fyuA } & \multirow{2}{*}{ Gene of the pesticin receptor of Yersinia } & \multirow{2}{*}{235} & F-ACCGTTATCGCCATTCTG \\
\hline & & & R-CTGTGAAGTCTGGGCATTAG \\
\hline \multicolumn{4}{|c|}{ Adhesins-related genes } \\
\hline \multirow{2}{*}{ рарА } & \multirow{2}{*}{ Genes encoding parts of the $\mathrm{P}$ pilus } & 374 & F-GCTCCAACTATTCCACAG \\
\hline & & 年 & R-TTCAGGGTATTAGCATCAC \\
\hline рарС & Genes encoding parts of the $\mathrm{P}$ pilus & 234 & F-GGGCGTGATAACGATTC \\
\hline & & & R-ATTTGCCAGCGGACTAC \\
\hline eqeA & Encoding outer membrane protein intimin & 827 & F-GCGTTACATTGACTCCC \\
\hline cant & 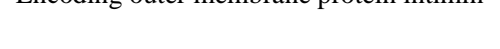 & $0<1$ & R-CATTGCTACCACCTTGC \\
\hline Fim A & Genes encodino narts of the F1 nilus & 352 & F-CAGGTTCGTACCGCATCG \\
\hline स & - & Suz & R-TCGCATCCGCATTAGCAG \\
\hline FimC & The Type 1 fimbrial adhesin & 337 & F-GCCGATGGTGTAAAGGAT \\
\hline & & & R-CCGTCAGGTAATAGGGTGT \\
\hline & & ins-related genes & \\
\hline vat & Toxin gene & 861 & F-TAAATGAGGTGGGCTGTG \\
\hline & & & R-AGGATGCCTCCGTAAACT \\
\hline sto1 & Shioa toxin 1 & 374 & F-GCCATTCGTTGACTACTT \\
\hline $\sin t$ & Jinga tusin 1 & $3 / 4$ & R-CTCATCAGATGCCATTCT \\
\hline$s+x 2$ & Shion toxin 2 & 240 & F-ACTGTCTGAAACTGCTCC \\
\hline $\operatorname{sen} 2$ & 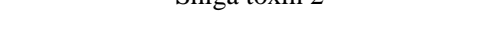 & $2+0$ & R-TGACATTCTGGTTGACTCT \\
\hline hlva & Transport gene of the hemolvsin oneron & 290 & F-TTGGGATACGCTGATAGG \\
\hline meghi & Н & & R-CACCCTTGACTAATAACTCG \\
\hline & & Other factors & \\
\hline$c o l V$ & ColV nroduction & 307 & F-ACGGATGCTCAGTTTCT \\
\hline & Sorv pivcuctiont & & R-TGTGCTTGGCGTCATAG \\
\hline$C O L B M$ & Colicins $\mathrm{B}$ and colicins $\mathrm{M}$ & 226 & F-GAGCCTGCTGTCACCСТT \\
\hline COIDIMI & Contins D dilu conicins la & $\angle \angle 0$ & R-GTTCAGATAATGCCCGATG \\
\hline
\end{tabular}

${ }^{\mathrm{a}} \mathrm{F}$ : forward; R: reverse. 
specific PCR products of expected sizes were revealed by agarose gel electrophoresis (Figure 1 and Figure 2). The sequences of these virulence-associated genes were determined and deposited with GenBank accession numbers, as shown in Table 3. Sequence analysis revealed that these 13 virulence-associated gene sequences detected in this study show high identity with those of published genes (Table 3).

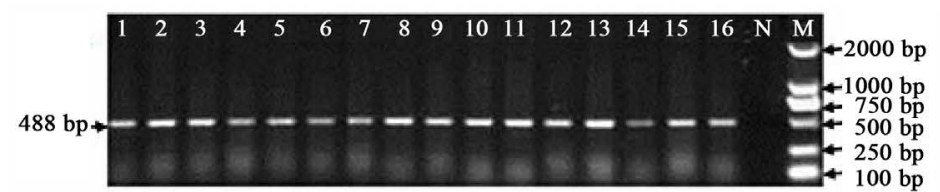

(a)

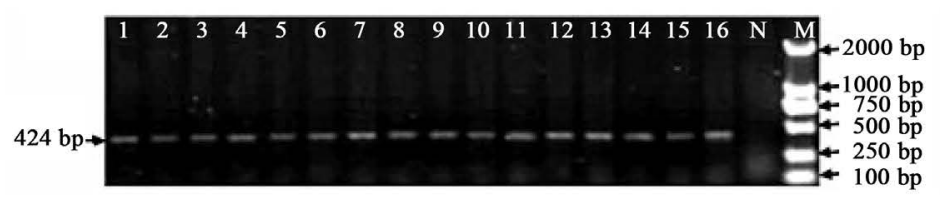

(b)

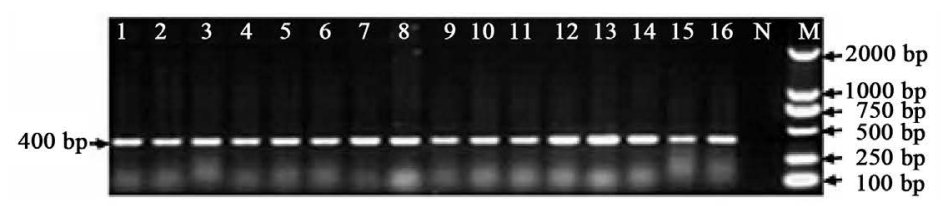

(c)

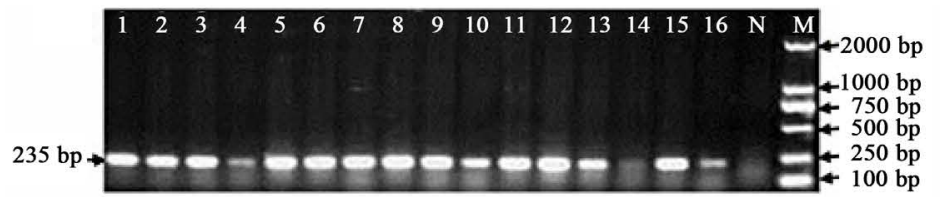

(d)

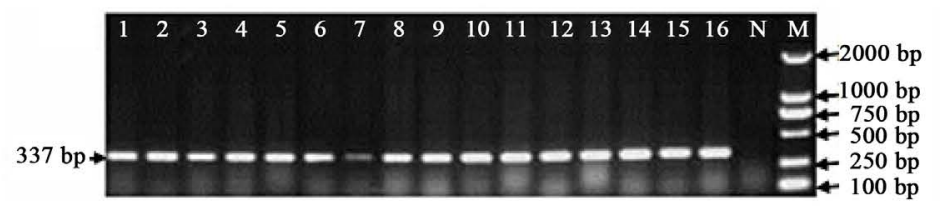

(e)

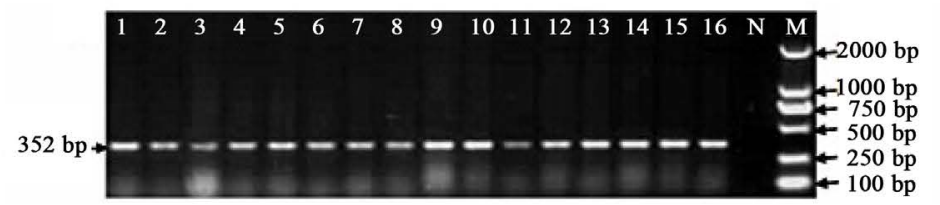

(f)

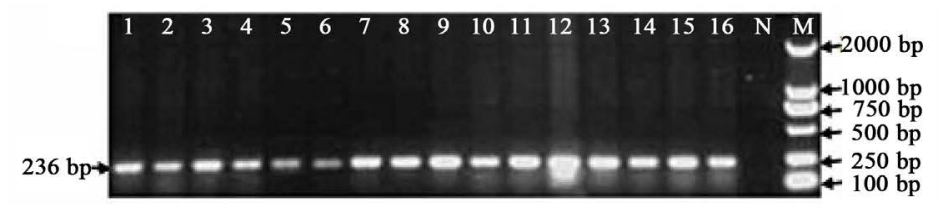

(g)

Figure 1. PCR amplification of 7 virulence-associated genes in E. coli strains. The specific PCR products of expected sizes were revealed by agarose gel electrophoresis. Representative electrophoretograms for PCR products amplified from each pathogen were shown. Lane M, DL 2000 marker; Lane N, Negative control. Panel (a), lanes 1 - 16: strains carried tsh; Panel (b) lanes 1 - 16: strains carried iutA; Panel (c), lanes 1 - 16: strains carried iucD; Panel (d), lanes 1 - 16: strains carried fyuA; Panel (e), lanes 1 - 16: strains carried FimC; Panel (f), lanes 1 - 16: strains carried FimA; Panel (g), lanes 1 - 16: strains carried irp2. 


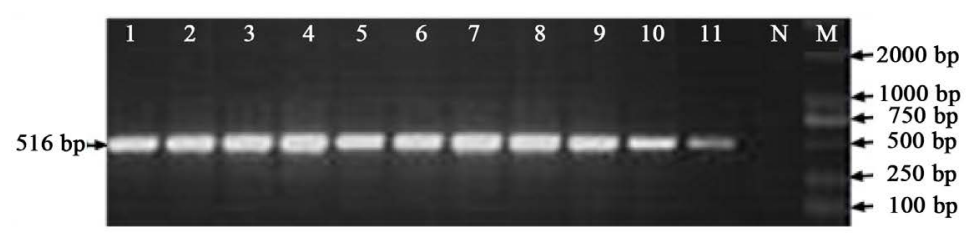

(a)

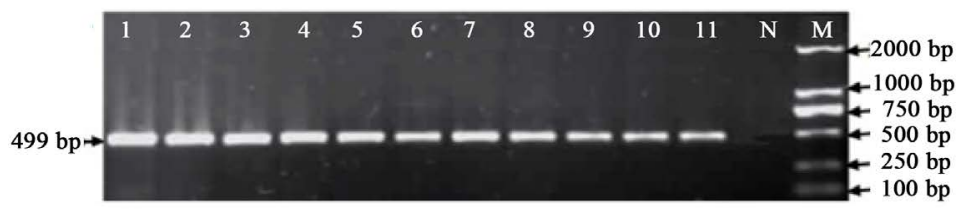

(b)

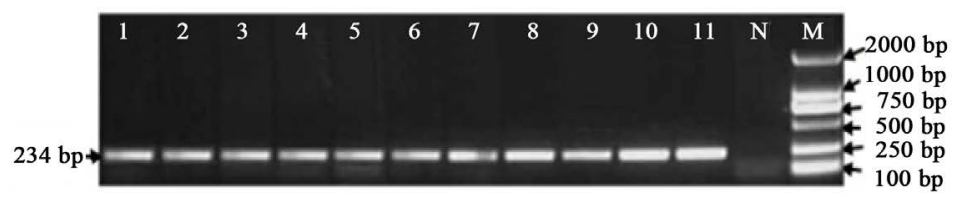

(c)

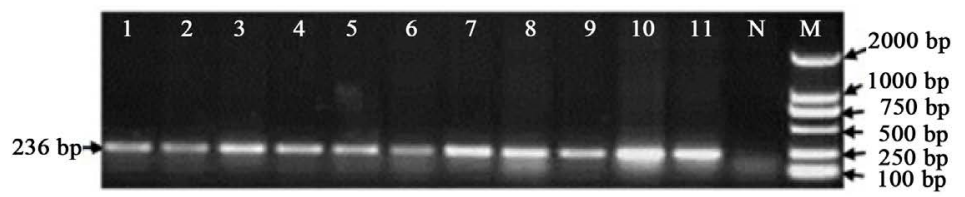

(d)

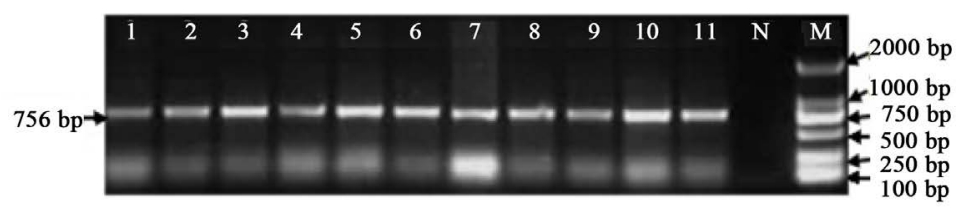

(e)

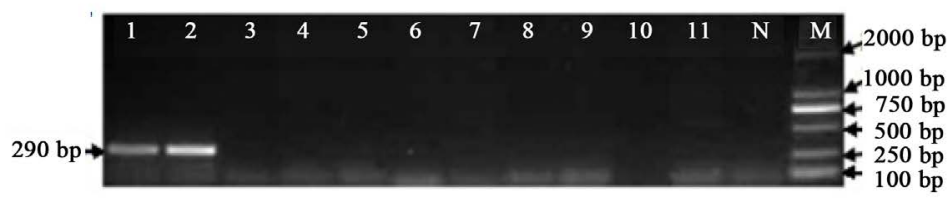

(f)

Figure 2. PCR amplification of 6 virulence-associated genes in $E$. coli strains. The specific PCR products of expected sizes were revealed by agarose gel electrophoresis. Representative electrophoretograms for PCR products amplified from each pathogen were shown. Lane M, DL 2000 marker; Lane N, Negative control. Panel (a), lanes 1 - 11: strains carried colBM; Panel (b), lanes 1 - 11: strains carried colV; Panel (c), lanes 1 - 11: strains carried papC; Panel (d), lanes 1 - 11: strains iucA; Panel (E), lanes 1 - 11: strains carried iss; Panel (f), lanes 1-2: strains carried hlyA; lanes 3 - 11, hlyA gene not detectable.

\subsection{Detection of 13 Virulence-Associated Genes in Pathogenic and Non-pathogenic Escherichia coli Strains}

Among 320 pathogenic and 50 non-pathogenic Escherichia coli strains examined, at least 2 to 13 genes were detected (Table 4). By examining 18 virulence-associated genes, the papA, vat, eaeA, st 1 , and st 22 genes were not detectable in this study. Among the 13 virulence-associated genes detected, the most prevalent is colBM (100\%) and the least one is hlyA (0.6\%) (Table 4).

Our results reveal that the number of virulence genes in 193 high pathogenic Escherichia coli strains was significantly higher than that of 127 intermediate and low pathogenic strains $(P<0.01)$. Furthermore, the detection rates of the virulence genes for irp2, fyuA, iutA, papC, iss, colV, and iucD in 291 high and intermediate pa- 
Table 3. Sequence homology of 13 virulence-associated genes in chicken $E$. coli strains compared with published sequences.

\begin{tabular}{cccc}
\hline $\begin{array}{c}\text { Virulence-associated } \\
\text { genes }\end{array}$ & $\begin{array}{c}\text { Isolate sequences } \\
\text { (accession no. })^{\mathrm{a}}\end{array}$ & $\begin{array}{c}\text { Published sequences } \\
\text { (accession no.) }\end{array}$ & $\begin{array}{c}\text { Percentage of sequence } \\
\text { homology (\%) }\end{array}$ \\
\hline irp2 & JX485631 & EU120030.1 & $98.9 \%-99.4 \%$ \\
fyuA & JX466849 & Z38064.1 & $99.1 \%-99.4 \%$ \\
iucA & JX466845 & X76100.1 & $98.5 \%-99.3 \%$ \\
iucD & JX466843 & NC009837.1 & $98.9 \%-99.1 \%$ \\
iutA & JX466848 & NC009837.1 & $99.2 \%-99.8 \%$ \\
FimA & JX466846 & NC000913.2 & $98.7 \%-99.4 \%$ \\
FimC & JX466847 & FJ866110.1 & $99.4 \%-99.6 \%$ \\
papC & JX485632 & HQ165752.1 & $98.4 \%-99.5 \%$ \\
hlyA & JX466842 & FM180012.1 & $99.2 \%-99.6 \%$ \\
iss & JX466844 & X52665.1 & $95.8 \%-99.6 \%$ \\
Tsh & JX466850 & AY280856.1 & $98.5 \%-99.2 \%$ \\
colV & KC447292 & AY545598.5 & $98.4 \%-99.3 \%$ \\
colBM & KC447290 & NC009837.1 & $98.4 \%-99.3 \%$ \\
\hline
\end{tabular}

${ }^{\mathrm{a}}$ Sequences determined in this study.

Table 4. Detection of virulence-associated genes in pathogenic and non-pathogenic strains.

\begin{tabular}{cccccc}
\hline \multirow{2}{*}{$\begin{array}{c}\text { Virulence } \\
\text { genes }\end{array}$} & $\begin{array}{c}\text { High pathogenic } \\
\text { strains } \\
(\mathrm{n}=193)\end{array}$ & $\begin{array}{c}\text { Intermediate } \\
\text { pathogenic strains } \\
(\mathrm{n}=98)\end{array}$ & $\begin{array}{c}\text { Low pathogenic } \\
\text { strains } \\
(\mathrm{n}=29)\end{array}$ & Total & $\begin{array}{c}\text { Non-pathogenic } \\
\text { strains } \\
(\mathrm{n}=50)\end{array}$ \\
\cline { 2 - 6 } irp2 & $87.6(169 / 193)$ & $44.9(44 / 98)$ & 0.0 & $66.6(213 / 320)$ & 0.0 \\
fyuA & $90.2(174 / 193)$ & $70.4(69 / 98)$ & $20.7(6 / 29)$ & $77.8(249 / 320)$ & 0.0 \\
iucA & $68.4(132 / 193)$ & $38.8(38 / 98)$ & $34.5(10 / 29)$ & $53.1(170 / 320)$ & $30(15 / 50)$ \\
iucD & $80.8(156 / 193)$ & $70.4(70 / 98)$ & $34.5(10 / 29)$ & $73.8(236 / 320)$ & $20(10 / 50)$ \\
iutA & $85.5(165 / 193)$ & $54.1(53 / 98)$ & $24.1(7 / 29)$ & $70.3(225 / 320)$ & $20(10 / 50)$ \\
FimA & $77.2(149 / 193)$ & $46.9(46 / 98)$ & $41.4(12 / 29)$ & $64.7(207 / 320)$ & $60(30 / 50)$ \\
FimC & $92.7(179 / 193)$ & $86.7(85 / 98)$ & $75.9(22 / 29)$ & $90.9(291 / 320)$ & $70(35 / 50)$ \\
papC & $31.1(60 / 193)$ & $4.1(4 / 98)$ & 0.0 & $20.0(64 / 320)$ & 0.0 \\
iss & $72.5(140 / 193)$ & $34.7(34 / 98)$ & $13.8(4 / 29)$ & $55.6(178 / 320)$ & 0.0 \\
hlyA & $1.0(2 / 193)$ & 0.0 & 0.0 & $0.6(2 / 320)$ & 0.0 \\
tsh & $83.4(161 / 193)$ & $29.6(29 / 98)$ & $27.6(8 / 29)$ & $61.8(198 / 320)$ & $16(8 / 50)$ \\
colV & $85.5(165 / 193)$ & $50.0(49 / 98)$ & 0.0 & $66.9(214 / 320)$ & 0.0 \\
colBM & $100.0(193 / 193)$ & $100.0(98 / 98)$ & $100.0(29 / 29)$ & $100.0(320 / 320)$ & $100(50 / 50)$ \\
\hline
\end{tabular}

thogenic Escherichia coli strains were significantly higher than that of 29 low pathogenic and non-pathogenic Escherichia coli strains $(P<0.01)$. The detection rate of the virulence genes for iucA, fimA, and tsh in intermediate and low pathogenic Escherichia coli strains shows no significant difference $(P>0.05)$. The detection rate of virulence genes for FimC and colBM in three different pathogenic Escherichia coli strains shows no significant difference $(P>0.05)$. Interestingly, the hemolysin hlyA gene was detected only in highly pathogenic chicken Escherichia coli. 


\subsection{Combinations of Virulence-Associated Genes and Their Correlations with Pathogenicity}

By examining 193 highly pathogenic Escherichia coli strains, it was found that all strains simultaneously carried at least 8 to13 virulence genes. As shown in Table 5, 14 strains (7.3\%) simultaneously carried 12 virulence genes, causing the death of 4 - 5 chickens within 24 hours; 85 strains (44\%) simultaneously carried 10 or 11 virulence genes, causing the death of 4 - 5 chickens within 72 hours. Among 13 virulence genes detected in 193 highly pathogenic Escherichia coli strains, a total of 51 different combinations of virulence-associated genes were observed. It was found that 134 of 193 (69.43\%) highly pathogenic Escherichia coli strains belonged to 10 predominant patterns as indicated in Table 5. The most prevalence of combination patterns of virulence-associated genes $(i r p 2+f y u A+i u c A+i u c D+i u t A+f i m A+f i m C+i s s+t s h+c o l V+\operatorname{colBM}$ and $r p 2+f y u A+$ $i u c A+i u c D+i u t A+f i m A+f i m C+p a p C+i s s+t s h+c o l V+c o l B M)$ in highly pathogenic Escherichia coli strains were $22.3 \%$ and $7.3 \%$, respectively (Table 5 ).

By examining 98 intermediate pathogenic Escherichia coli strains, 12 virulence genes were detected in these strains which simultaneously carried at least 5-8 virulence genes. In the present study, 36 different combination patterns from these 12 virulence genes in 98 intermediate pathogenic Escherichia coli strains were found and $60.2 \%$ (59/98) of them belonged to the 7 predominant patterns (Table 5). The most prevalence combination patterns of virulence-associated genes $(\operatorname{irp} 2+$ fyu $A+i u t A+f i m C+i s s+\operatorname{colV}+\operatorname{colBM}$ and irp $2+f y u A+$ $i u c D+f i m A+f i m C+\operatorname{col} V+\operatorname{colBM})$ in intermediate pathogenic Escherichia coli strains were $13.2 \%$ and $10.2 \%$, respectively (Table 5).

By assessing 29 low pathogenic Escherichia coli strains, 9 virulence genes were detected in these strains which simultaneously carry at least 2 to 4 virulence genes. Twelve different combinations from these 9 virulence genes were observed, and 62\% (18/29) of them belonged to 4 predominant patterns (Table 6). The most prevalence combination patterns of virulence-associated genes (iucD $+i u t A+$ fim $C+\operatorname{colBM}$ and fimA + fimC + colBM ) in low pathogenic Escherichia coli strains were $20.7 \%$ and $17.2 \%$, respectively (Table 6 ). Furthermore, there are 7 virulence genes that were detected in 50 non-pathogenic Escherichia coli strains, and each carries at least 2 to 4 virulence genes. Four different combinations from 7 virulence genes were seen, and 70\% (14/20) of them belong to 2 predominant patterns (Table 6). The major combination patterns of virulence-associated genes $($ colBM + fimA and colBM + fimC + tsh $)$ in non-pathogenic Escherichia coli strains were $20.7 \%$ and $30 \%$, respectively (Table 6).

Table 5. The predominant patterns of virulence genes in 193 high pathogenic and 98 intermediate pathogenic strains.

\begin{tabular}{|c|c|c|c|}
\hline \multicolumn{2}{|l|}{193 high pathogenic strains } & \multicolumn{2}{|l|}{98 intermediate pathogenic strains } \\
\hline Combination patterns & $\begin{array}{l}\text { Detection (\%) } \\
\text { (No. of positive } \\
\text { strains/No. of } \\
\text { total strains) }\end{array}$ & Combination patterns & $\begin{array}{l}\text { Detection (\%) } \\
\text { (No of positive } \\
\text { strains/No. of } \\
\text { total strains) }\end{array}$ \\
\hline $\begin{array}{l}\text { colBM }+i r p 2+f y u A+i u c A+i u c D+ \\
i u t A+f i m A+f i m C+t s h+c o l V+i s s\end{array}$ & $22.3(43 / 193)$ & $\operatorname{colBM}+i r p 2+f y u A+i u t A+f i m C+i s s+\operatorname{col} V$ & $13.2(13 / 98)$ \\
\hline $\begin{array}{l}\text { colBM }+i r p 2+f y u A+i u c A+i u c D+i u t A \\
+ \text { fim } A+f i m C+t s h+c o l V+p a p C+i s s\end{array}$ & $7.3(14 / 193)$ & $\operatorname{colBM}+i r p 2+f y u A+i u c D+f i m A+f i m C+\operatorname{col} V$ & $10.2(10 / 98)$ \\
\hline $\begin{aligned} \text { colBM } & +i r p 2+f y u A+i u c A+i u c D+i u t A \\
& + \text { fim } A+f i m C+t s h+\operatorname{col} V\end{aligned}$ & $6.7(13 / 193)$ & $\operatorname{colBM}+i r p 2+f y u A+i u c A+i u c D+f i m C$ & $8.2(8 / 98)$ \\
\hline $\begin{array}{c}\text { colBM }+i r p 2+f y u A+i u c A+i u c D+f i m A \\
+ \text { fimC }+ \text { papC }+i s s+t s h+c o l V\end{array}$ & $6.2(12 / 193)$ & $c o l B M+i u c D+i u t A+f i m C+t s h$ & $8.2(8 / 98)$ \\
\hline $\begin{array}{c}\text { colBM }+\operatorname{irp} 2+f y u A+i u t A+f i m A+f i m C \\
+ \text { papC }+ \text { tsh }+ \text { colV }\end{array}$ & $5.7(11 / 193)$ & $c o l B M+f y u A+i u c A+i u c D+f i m A+f i m C+c o l V$ & $7.1(7 / 98)$ \\
\hline $\begin{aligned} \operatorname{colBM} & +\operatorname{irp} 2+f y u A+i u t A+i u c D+f i m A \\
& + \text { fimC }+i s s+t s h+\operatorname{col} V\end{aligned}$ & $4.7(9 / 193)$ & $\operatorname{colBM}+f y u A+i u c D+f i m A+f i m C+i s s$ & $7.1(7 / 98)$ \\
\hline $\begin{array}{c}\text { colBM }+i r p 2+f y u A+i u t A+i u c D+\text { fimA } \\
+ \text { fimC }+ \text { iss }+ \text { colV }\end{array}$ & $4.7(9 / 193)$ & $\operatorname{colBM}+i r p 2+f y u A+i u c A+f i m C$ & $6.1(6 / 98)$ \\
\hline $\begin{array}{c}\text { colBM }+i r p 2+f y u A+i u c A+i u c D+i u t A \\
+ \text { fimC }+ \text { pap } C+i s s+t s h+c o l V\end{array}$ & $4.1(8 / 193)$ & & \\
\hline $\begin{array}{c}\text { colBM }+i r p 2+f y u A+i u c D+f i m A+f i m C \\
+i s s+t s h+c o l V\end{array}$ & $4.1(8 / 193)$ & & \\
\hline
\end{tabular}


Table 6. The predominant patterns of virulence genes in 29 low pathogenic and 20 non-pathogenic strains.

\begin{tabular}{|c|c|c|c|}
\hline \multicolumn{2}{|c|}{29 low pathogenic strains } & \multicolumn{2}{|c|}{50 non-pathogenic strains } \\
\hline Combination patterns & $\begin{array}{c}\text { Detection (\%) } \\
\text { (No. of positive strains/No. } \\
\text { of total strains) }\end{array}$ & Combination patterns & $\begin{array}{c}\text { Detection (\%) } \\
\text { (No. of positive strains/No. } \\
\text { of total strains) }\end{array}$ \\
\hline $\operatorname{colBM}+f i m C+i u c D+i u t A$ & $20.7(6 / 29)$ & $\operatorname{colBM}+f i m C+F i m A+i u c A$ & $20.0(10 / 50)$ \\
\hline $\operatorname{colBM}+f y u A+f i m C+t s h$ & $10.3(3 / 29)$ & $\operatorname{colBM}+\operatorname{fim} A+i u c D+i u t A$ & $10.0(5 / 50)$ \\
\hline $\operatorname{colBM}+\operatorname{fimA}+$ fimC & $17.2(5 / 29)$ & $\operatorname{colBM}+f i m C+t s h$ & $30.0(15 / 50)$ \\
\hline $\operatorname{colBM}+$ fimA $+t s h$ & $13.8(4 / 29)$ & $\operatorname{colBM}+$ fimA & $40.0(20 / 50)$ \\
\hline
\end{tabular}

Among 320 Escherichia coli strains examined, the detection rate of high pathogenicity island genes (irp2, fyuA, and colV) in high pathogenic strains were significantly higher than those in low and non-pathogenic strains $(P<0.01)$ (Table 7). Nine virulence-associated genes irp2, fyuA, iucA, iucD, iutA, papC, iss, tsh, and colV were significantly more often detected in high and intermediate pathogenic E. coli strains (Table 7). Furthermore, our results revealed that the combination patterns from five or more than five genes in high and intermediate pathogenic $E$. coli strains were highly correlated with its pathogenicity $(P<0.01)$.

\section{Discussion}

In recent years, virulence-related genes or pathogenicity island were found in many pathogenic bacteria, and this may be related to the evolution of bacterial virulence. For example, high pathogenicity island (HPI) is firstly discovered in Yersinia, but it also widely exists in Escherichia coli of human, pigs, cattle and rabbit [15]-[20]. Both irp2 and fyuA genes are closely associated with HPI, and can be used as the marker in HPI detection [21]. A previous report by Hu et al. suggested that the detection rate of irp2 in pathogenic Escherichia coli of human was $10.0 \%$ [22]. More recently, Smith et al. reported that the detection rate of fyuA in pathogenic Escherichia coli strains of pigs was $15.9 \%$ [23]. In the present study, the higher detection rates of genes for irp2 and fyuA (66.6\% and 77.8\%) in chicken pathogenic Escherichia coli were observed. Furthermore, our results reveal that chicken pathogenic Escherichia coli strains simultaneously carried irp2 and fyuA genes were up to 60.3\% (193/320). The detection rate of these HPI gene combination in highly chicken pathogenic strains were $80.8 \%$ (156/193), showing significant difference $(P<0.01)$ than that in intermediate $(37 / 98,37.8 \%)$ and low $(0 / 29,0 \%)$ pathogenic strains. The present study provides evidences suggesting that HPI pathogenicity islands and its related genes are closely associated with the pathogenicity of chicken Escherichia coli strains.

Pilus is the main virulent factor that is involved in adhesion of pathogenic Escherichia coli to the host cells, including types I and P pilus. Previous study suggested that type I pili existed mainly in pathogenic Escherichia coli strains, and it existed more widely in chicken pathogenic Escherichia coli than P pili [24]. Both FimA and FimC genes are responsible for encoding essential protein of biosynthesis process of type I pili while PapA and papC are important functional genes of type $\mathrm{P}$ pili that encode the main component proteins of type $\mathrm{P}$ pili. A previous study by Galli et al. reported that the detection rate of FimA gene was $92.8 \%$ in bovine E. coli strains [25]. It was also reported that $60.0 \%$ of FimC gene were detected in chicken E. coli among isolates tested [26]. In the present study, the detection rate of FimA gene (64.4\%) is lower than that reported by Galli et al. [25] whereas FimC gene (89.7\%) is higher than that reported by Wang et al. 2004. A previous investigation found that $6.5 \%$ of chicken pathogenic Escherichia coli isolates carried papC gene [27]. It is interesting to note that the higher detection rate of this gene (15.9\%) than that reported by Jin et al. [27] was seen, but the papA gene in chicken pathogenic Escherichia coli strains was not detected in all tested strains.

ColV plasmid widely exists in avian pathogenic Escherichia coli strains which correlate with the strain virulence. It contains a variety of virulence genes, such as gas bacillus gene iucA, iucD and iutA, Iss protein gene iss, hemolysin A gene hlyA and temperature-sensitive hemagglutinin gene tsh. They are important virulence genes related to pathogenicity of Escherichia coli, and mainly exist in the ColV plasmid [28]. ColBM plasmid is a newly discovered E. coli virulence factor, which may evolve from ColV plasmid, and it possesses virulence genes that are similar to ColV [29]. Our study reveals that the detection rate of ColBM gene (100\%) in chicken pathogenic Escherichia coli strains is much higher than that of ColV gene (66.6\%). The reason why the detection rate showing big difference needs to be further studied. 
Table 7. Eight of the highest rate of virulence gene combinations in high pathogenic and intermediate pathogenic strains.

\begin{tabular}{|c|c|c|c|}
\hline \multicolumn{2}{|l|}{193 high pathogenic strains } & \multicolumn{2}{|c|}{98 intermediate pathogenic strains } \\
\hline Combination patterns & $\begin{array}{l}\text { Detection (\%) } \\
\text { (No of positive } \\
\text { strains/No. of } \\
\text { total strains) }\end{array}$ & Combination patterns & $\begin{array}{l}\text { Detection (\%) } \\
\text { (No of positive } \\
\text { strains/No. } \\
\text { of total strains) }\end{array}$ \\
\hline $\operatorname{colBM}+\operatorname{irp} 2+f y u A+f i m C+\operatorname{col} V$ & $82.9(160 / 193)$ & $\operatorname{colBM}+$ fimC & $86.7(85 / 98)$ \\
\hline $\operatorname{colBM}+i r p 2+f y u A+f i m C+\operatorname{colV}+i u t A$ & $82.4(159 / 193)$ & $c o l B M+f i m C+f y u A$ & $68.4(67 / 98)$ \\
\hline $\operatorname{colBM}+i r p 2+f y u A+f i m C+\operatorname{colV}+t s h$ & $81.3(157 / 193)$ & $c o l B M+f i m C+i u c D$ & $66.3(65 / 98)$ \\
\hline $\operatorname{colBM}+\operatorname{irp} 2+f y u A+f i m C+\operatorname{col} V+i u c D$ & $79.3(153 / 193)$ & $\operatorname{colBM}+f i m C+f y u A+i u c D$ & $60.2(59 / 98)$ \\
\hline $\operatorname{colBM}+\operatorname{irp} 2+f y u A+f i m C+\operatorname{col} V+f i m A$ & $76.2(147 / 193)$ & $\operatorname{colBM}+$ fimC $+i u t A$ & $52.0(51 / 98)$ \\
\hline $\operatorname{colBM}+i r p 2+f y u A+f i m C+\operatorname{colV}+i s s$ & $71.5(138 / 193)$ & $\operatorname{colBM}+\mathrm{fimC}+\operatorname{col} V$ & $50.0(49 / 98)$ \\
\hline $\operatorname{colBM}+i r p 2+f y u A+f i m C+\operatorname{col} V+i u c A$ & $68.4(132 / 193)$ & $\operatorname{colBM}+$ fimC $+i r p 2$ & $43.9(43 / 98)$ \\
\hline $\operatorname{colBM}+i r p 2+f y u A+f i m C+\operatorname{col} V+i u t A+i u c D$ & $72.0(139 / 193)$ & $\operatorname{colBM}+\operatorname{fim} C+f i m A$ & 43.9(43/98) \\
\hline
\end{tabular}

At present, studies mainly focus on the types and distribution patterns of virulence-related genes [27]. In the present study, we show the correlations between the numbers and combination patterns of virulence-related genes and their pathogenicity in pathogenic E. coli strains. The number and combination patterns of virulencerelated genes detected in highly and intermediate chicken pathogenic strains showed more significant difference than those in low and non-pathogenic strains $(P<0.01)$. Our results reveal that there are at least 8 to 13 virulence-related genes and 51 different combination patterns from these genes in the highly pathogenic chicken $E$. coli strains and at least 5 to 8 virulence-related genes and 36 different combination patterns from the genes in the intermediate pathogenic chicken $E$. coli strains, suggesting that the number and combination patterns of these virulence-related genes are significantly correlated with the pathogenicity $(P<0.01)$. This study provides an important insight into the correlation between the number and combination patterns of virulence-associated genes and E. coli pathogenicity.

\section{Acknowledgements}

This work was supported by grants from the National Science Foundation of China (grant No. 31272577) and the Ministry of Education, Taiwan, under the ATU plan.

\section{References}

[1] Lutful Kabir, S.M. (2010) Avian Colibacillosis and Salmonellosis: A Closer Look at Epidemiology, Pathogenesis, Diagnosis, Control and Public Health Concerns. International Journal of Environmental Research and Public Health, 7, 89-114. http://dx.doi.org/10.3390/ijerph7010089

[2] Vandekerchove, D., Vandemaele, F., Adriaensen, C., Zaleska, M., Hernalsteens, J.P., De Baets, L., Butaye, P., Van Immerseel, F., Wattiau, P., Laevens, H., Mast, J., Goddeeris, B. and Pasmans, F. (2005) Virulence-Associated Traits in Avian Escherichia coli: Comparison between Isolates from Colibacillosis-Affected and Clinically Healthy Layer Flocks. Veterinary Microbiology, 108, 75-87. http://dx.doi.org/10.1016/j.vetmic.2005.02.009

[3] Cheng, D.R., Sun, H.C., Xu, J.S. and Gao, S. (2006) Prevalence of LEE and HPI Pathogenicity Islands of Escherichia coli Isolates from Weaned Piglets in China. Acta Microbiologica Sinica, 46, 368-372.

[4] Yu, J. and Kaper, J.B. (1992) Cloning and Characterization of the eae Gene of Enterohaemorrhagic Escherichia coli O157:H7. Molecular Microbiology, 6, 411-417. http://dx.doi.org/10.1111/j.1365-2958.1992.tb01484.X

[5] Horne, S.M., Pfaff-McDonough, S.J., Giddings, C.W. and Nolan, L.K. (2000) Cloning and Sequencing of the iss Gene from a Virulent Avian Escherichia coli. Avian Diseases, 44, 179-184. http://dx.doi.org/10.2307/1592522

[6] Forman, S., Nagiec, M.J., Abney, J., Perry, R.D. and Fetherston, J.D. (2007) Analysis of the Aerobactin and Ferric Hydroxamate Uptake Systems of Yersinia pestis. Microbiology, 153, 2332-2341. http://dx.doi.org/10.1099/mic.0.2006/004275-0

[7] Xia, M.Y., Tang, W. and Yue, H. (2010) Progress on Virulence-Associated Factors ColBM and RfaH in Escherichia 
coli. Progress in Veterinary Medicine, 31, 86-89.

[8] Carniel, E., Guilvout, I. and Prentice, M. (1996) Characterization of a Large Chromosomal "High-Pathogenicity Island” in Biotype 1B Yersinia enterocolitica. Journal of Bacteriology, 178, 6743-6751.

[9] Provence, D.L. and Curtiss, R.R. (1994) Isolation and Characterization of a Gene Involved in Hemagglutination by an Avian Pathogenic Escherichia coli strain. Infection and Immunity, 62, 1369-1380.

[10] Cloud, S.S., K.Rosenberger, J., Fries, P.A., Wilson, R.A. and Odor, E.M. (1985) In Vitro and in Vivo Characterization of Avian Escherichia coli. I. Serotypes, Metabolic Activity, and Antibiotic Sensitivity. Avian Disease, 29, 1084-1093. http://dx.doi.org/10.2307/1590463

[11] Rosenberger, J.K., Fries, P.A., Cloud, S.S. and Wilson, R.A. (1985) In Vitro and in Vivo Characterization of Avian Escherichia coli. II. Factors Associated with Pathogenicity. Avian Diseases, 29, 1094-1107. http://dx.doi.org/10.2307/1590464

[12] Rosenberger, J.K., Fries, P.A. and Cloud, S.S. (1985) In Vitro and in Vivo Characterization of Avian Escherichia coli. III. Immunization. Avian Diseases, 29, 1108-1117. http://dx.doi.org/10.2307/1590465

[13] Janben, T., Schwarz, C., Preikschat, P., Voss, M., Philipp, H.C. and Wieler, L.H. (2001) Virulence-Associated Genes in Avian Pathogenic Escherichia coli (APEC) Isolated from Internal Organs of Poultry Having Died from Colibacillosis. International Journal of Medical Microbiology, 291, 371-378. http://dx.doi.org/10.1078/1438-4221-00143

[14] Johnson, J.R. and Stell, A.L. (2000) Extended Virulence Genotypes of Escherichia coli Strains from Patients with Urosepsis in Relation to Phylogeny and Host Compromise. Journal of Infectious Diseases, 181, 261-272. http://dx.doi.org/10.1086/315217

[15] Sperandio, V., Kaper, J.B., Bortolini, M.R., Neves, B.C., Keller, R. and Trabulsi, L.R. (1998) Characterization of the Locus of Enterocyte Effacement (LEE) in Different Enteropathogenic Escherichia coli (EPEC) and Shiga-Toxin Producing Escherichia coli (STEC) Serotypes. FEMS Microbiology Letters, 164, 133-139. http://dx.doi.org/10.1111/j.1574-6968.1998.tb13078.x

[16] Bach, S., de Almeida, A. and Carniel, E. (2000) The Yersinia High-Pathogenicity Island Is Present in Different Members of the Family Enterobacteriaceae. FEMS Microbiology Letters, 183, 289-294.

http://dx.doi.org/10.1111/j.1574-6968.2000.tb08973.x

[17] Carniel, E. (2001) The Yersinia High-Pathogenicity Island: An Iron-Uptake Island. Microbes Infection, 3, $561-569$. http://dx.doi.org/10.1016/S1286-4579(01)01412-5

[18] Clermont, O., Bonacorsi, S. and Bingen, E. (2001) The Yersinia High-Pathogenicity Island Is Highly Predominant in Virulence-Associated Phylogenetic Groups of Escherichia coli. FEMS Microbiology Letters, 196, 153-157. http://dx.doi.org/10.1111/j.1574-6968.2001.tb10557.x

[19] Jores, J., Rumer, L., Kiessling, S., Kaper, J.B. and Wieler, L.H. (2001) A Novel Locus of Enterocyte Effacement (LEE) Pathogenicity Island Inserted at pheV in Bovine Shiga Toxin-Producing Escherichia coli Strain O103:H2. FEMS Microbiology Letters, 204, 75-79. http://dx.doi.org/10.1111/j.1574-6968.2001.tb10866.x

[20] Penteado, A.S., Ugrinovich, L.A., Blanco, J., Blanco, M., Blanco, J.E., Mora, A., Andrade, J.R., Correa, S.S. and Pestana de Castro, A.F. (2002) Serobiotypes and Virulence Genes of Escherichia coli Strains Isolated from Diarrheic and Healthy Rabbits in Brazil. Veterinary Microbiology, 89, 41-51. http://dx.doi.org/10.1016/S0378-1135(02)00148-7

[21] Rodriguez-Siek, K.E., Giddings, C.W., Doetkott, C., Johnson, T.J. and Nolan L.K. (2005) Characterizing the APEC Pathotype. Veterinary Research, 36, 241-256. http://dx.doi.org/10.1051/vetres:2004057

[22] Hu, Y.X., Ou-Yang, D.M., Yu, X.F., Li, M.L., Liu, Q.T. and He, Z.X. (2008) Analysis of Partial Sequence of Enteropathogenic Escherichia coli Strains with Yersinia HPI irp2. Journal of Chinese Infection Control, 7, 167-169.

[23] Smith, M.G., Jordan, D., Chapman, T.A., Chin, J.J., Barton, M.D., Do, T.N., Fahy, V.A., Fairbrother, J.M. and Trott, D.J. (2010) Antimicrobial Resistance and Virulence Gene Profiles in Multi-Drug Resistant Enterotoxigenic Escherichia coli Isolated from Pigs with Post-Weaning Diarrhoea. Veterinary Microbiology, 145, 299-307. http://dx.doi.org/10.1016/j.vetmic.2010.04.004

[24] La Ragione, R.M., Mclaren, I.M., Foster, G., Cooley, W.A. and Woodward, M.J. (2002) Phenotypic and Genotypic Characterisation of Avian Escherichia coli O86:K61 Isolates Possessing a Gamma-Like Intimin. Applied and Environmental Microbiology, 68, 4932-4942. http://dx.doi.org/10.1128/AEM.68.10.4932-4942.2002

[25] Galli, L., Miliwebsky, E., Irino, K., Leotta, G. and Rivas, M. (2010) Virulence Profile Comparison between LEENegative Shiga Toxin-Producing Escherichia coli (STEC) Strains Isolated from Cattle and Humans. Veterinary Microbiology, 143, 307-313. http://dx.doi.org/10.1016/j.vetmic.2009.11.028

[26] Wang, Y.J., Fan, C. and Li, Y.J. (2004) The Relation between fimC Gene and Pathogenicity of Avian Escherichia coli. Acta Veterinaria et Zootechnica Sinica, 35, 680-684.

[27] Jin, W.J., Zheng, Z.M., Zhang, Y.Z., Qin, A.J., Shao, H.X., Liu, Y.L., Wang, J. and Wang, Q.D. (2008) Distribution of 
Virulence-Associated Genes of Avian Pathogenic Escherichia coli Isolates in China. Agricultural Science in China, 7, 1511-1515. http://dx.doi.org/10.1016/S1671-2927(08)60410-1

[28] Johnson, T.J., Siek, K.E., Johnson, S.J. and Nolan, L.K. (2006) DNA Sequence of a ColV Plasmid and Prevalence of Selected Plasmid-Encoded Virulence Genes among Avian Escherichia coli Strains. Journal of Bacteriology, 188, 745-758. http://dx.doi.org/10.1128/JB.188.2.745-758.2006

[29] Johnson, T.J., Johnson, S.J. and Nolan, L.K. (2006) Complete DNA Sequence of a ColBM Plasmid from Avian Pathogenic Escherichia coli Suggests That It Evolved from Closely Related ColV Virulence Plasmids. Journal of Bacteriology, 188, 5975-5983. http://dx.doi.org/10.1128/JB.00204-06 